\title{
Modified Iterated Extended Kalman Filter for Mobile Cooperative Tracking System
}

\author{
Rafina Destiarti A., Prima Kristalina, Amang Sudarsono \\ Department of Electrical Engineering, Magister Program of Engineering Technology \\ Politeknik Elektronika Negeri Surabaya (PENS) \\ Kampus PENS, Jalan Raya ITS, Sukolilo 60111, Surabaya, Indonesia \\ E-mail: finaa@pasca.student.pens.ac.id, \{prima,amang\}@pens.ac.id
}

\begin{abstract}
Tracking a mobile node using wireless sensor network (WSN) under cooperative system among anchor node and the mobile node, has been discussed in this work, interested to the indoor positioning applications. Developing an indoor location tracking system based on received signal strength indicator (RSSI) of WSN is considered cost effective and the simplest method. The suitable technique for estimating position out of RSSI measurements is the extended Kalman filter (EKF) which is especially used for nonlinear data as RSSI. In order to reduce the estimated errors from EKF algorithm, this work adopted forward data processing of the EKF algorithm to improve the accuracy of the filtering output, its called iterated extended Kalman filter (IEKF). However, using IEKF algorithm should know the stopping criterion value that is influenced to the maximum number iterations of this system. The number of iterations performed will be affected to the computation time although it can improve the estimation position. In this paper, we propose modified IEKF for mobile cooperative tracking system within only 4 iterations number. The illustrated results using RSSI measurements and simulation in MATLAB show that our proposed method has the capability to reduce error estimation percentage up to $19.3 \%$, with MSE (mean square error) $0.88 \mathrm{~m}$ compared with a conventional IEKF algorithm with MSE $1.09 \mathrm{~m}$. The time computation performance of our proposed method achieved in 3.55 seconds which is better than adding more iteration process.
\end{abstract}

Keywords — mobile cooperative tracking; WSN; RSSI; EKF; IEKF; modified IEKF

\section{INTRODUCTION}

Recently, there have been increasing interest in localization and tracking system in the indoor environment. There are many potential applications such as guidance of people in touring exhibitions, the monitoring of the patient in the hospital, monitoring some moving packages in a warehouse, or monitoring our pets, child and etc. in multistorey building [1]. Localization is the process of estimating or determining the location of the object which is applied to the sensor node in wireless sensor network (WSN) [2]. Another application of WSN is target tracking system that can be viewed as a sequential localization. The target tracking system is estimating directly the object which is requiring a real-time location estimation algorithm [3].

Typically, there are several measurement types of positioning methods, depending on obtaining the distance estimation between two nodes. The methods are RSSI (Received Signal Strength Indicators), AOA (Angle of Arrival), TDOA (Time Difference of Arrival) and TOA (Time of Arrival) [1]-[2], [4]. Although TOA and AOA have more accurate estimation compared to the other methods, this method require high-cost hardware which is making difficult implementation in some application [3]. RSSI-based positioning methods are used as far as they require no complex measurement hardware which is measured the power received from the transmitted radio signal antenna [5].

The mobile node needs to obtain the location estimation from multiple fixed reference node in mobile target tracking scenario. In forwarding the gathered tracking data from deployed node to a central observation point of the target node, the mobile node can communicate peer to peer with the nearest reference node using cooperative system [6]. When the mobile node is moving to different position, it will be communicated or received RSSI data from the other reference node based on its maximum coverage [7]. The position is obtained using trilateration method which is formed by intersection circle from three nearest distance of anchor nodes [4]-[6], [8].

RSSI-based localization method which is only used trilateration methods do not fulfil the accuracy requirements for being used in the indoor tracking system. This is due to the varied RSSI data depend on propagation effect such as shadowing, multipath fading, additive noise and some 
obstacles or materials of the indoor environment [9], [10]. This condition makes RSSI-based localization method using addition algorithm to linearize it. Kalman filter and its nonlinear extension, i.e. extended Kalman filter (EKF) provide a feasible solution to mitigate propagation effects and therefore it improves the accuracy of mobile target tracking in the indoor wireless environment [4], [9]. The EKF performs well for systems with mildly nonlinear functions, but if the measurement data is strongly nonlinear (such as tracking system), the performance of the filter deteriorates [11]. In such situation, the iterated extended Kalman filter (IEKF) achieves better consistency and accuracy compared to the EKF [1]1, [12]. The idea of IEKF is to improve the estimation by local iteration or forward data processing from the previous output estimation that is EKF algorithm. The iterations are usually stopped when there is no significant change in consecutive iterations or other criteria such as a maximum number iterations are met [11]. However, the time computation of IEKF is even slower than EKF, which limits its applications. In this paper, we investigate the role of iterations of IEKF in the mobile cooperative tracking system and then propose modified IEKF algorithm. We also extend our previous work [7] which is only used EKF algorithm for the mobile cooperative tracking system. The contribution of this paper is to propose another design system for mobile cooperative tracking that used modified IEKF algorithm to reduce the error estimation at the complex propagation environment. IEKF algorithm will filter the error data for each maximum coverage area of anchor nodes and also adopted the cluster-based system for grouping the various PLE into one area.

The fluctuative reduction of estimation result from the IEKF algorithm for each iteration will make the complicated system in determining the maximum number iterations of IEKF. Approaching the average result for each iteration and having a selection process to obtain the best result for all iterations process also conducted to this system. Modified IEKF which is proposed in this paper will be compared with the original EKF and conventional EKF for the mobile cooperative tracking system. Using mean square error (MSE) can be measured the error reduction of position estimation and also time computation speed which are influenced by the nodes hardware performance for calculating estimation result. Theoretical analysis will be given to prove the consistency, accuracy, and the scalability will be considered, as well. To demonstrate the performance of modified IEKF algorithm, mobile cooperative tracking system simulation using Matlab will be presented which is used the RSSI measurement data in a real scenario. Experimental results show that compared to original EKF and conventional IEKF, modified IEKF performs a more accurate estimate and also have almost same time computation with conventional IEKF.

The remainder of the paper is organized as follows: The related work was discussed in Section II, which were involved the basic equations of conventional IEKF with detailed theoretical analysis. The proposed algorithm for modified IEKF algorithm also in Section II. Both simulated and experimental results will be presented to verify the proposed algorithm in Section III. Finally, we draw the conclusion in Section IV.

\section{MATERIAL AND METHOD}

In this section, we describe the adopted algorithms which are used in the mobile cooperative tracking system. Those are trilateration algorithm which is used to obtain mobile node position based on intersection from three nearest distance of anchor nodes, EKF algorithm which is used to improve the mobile node estimation based on trilateration algorithm result, and iterated EKF based on the output of EKF to improve its performance.

\section{A. Related Work}

The utility of WSN for many applications is improved if the locations of the nodes especially in the indoor environment can be tracked based on the measurements between node communication. There are many measurements parameter which can be used for determining the position of the node. Those are estimated based on the RSSI measurement, TOA (time of arrival or time difference of arrival [1], [2], [4], [13]. Many applications, such as tracking the package in warehouse require the deployment of mobile ad hoc networks. Cooperative tracking is a relatively recent concept that attempts cooperative communication between mobile and anchor nodes. There are many researches which have considered cooperative tracking, i.e. using the Bayesian in the framework for cooperative tracking of nodes, which allows accurate tracking over large areas and using only a small number of anchor nodes [4], [13]. The other researchers used the cooperative localization beyond the range of the fixed tracking device which was called anchor nodes. Using cooperative tracking system allows the participating devices or node of this system come together to aid in the determination of location devices which otherwise would be out of scope [5], [6], [13].

Accuracy is the main challenge of the tracking system application especially for the indoor environment which was not covered by GPS (global positioning system) signal. Complex propagation characteristics, such as within buildings, obstacle, the material of building and the propagation range of the radio signal is short relative to the area to be covered due to the high path loss exponent value. Those problems make the tracking system require algorithm addition to linearize it which was included in non-linear condition. In this field, Kalman filter (KF) is able to achieve the optimal state estimation. However, it is not suitable for nonlinear systems. The best-known algorithm to solve the problem of nonlinear filtering is the extended Kalman filter (EKF) [1], [4], [7], [12]-[15]. This filter is based on the linearizing principle of the measurements data and evolution models using Taylor series expansions. RSSI positioning system which is influenced by path loss exponent value, have a non-linear characteristic, especially for the indoor environment. There are many researchers that have been modelled the propagation loss for determining the path loss exponent (PLE) value. In an RSSI positioning system, PLE is the parameter for converting RSSI to the distance estimation. The fluctuative data from RSSI will make the various value of PLE. The various number of PLE can be partitioned into some clusters based on characteristic environment and the position of the node. Cluster-based PLE areas from [16], can reduce the error performance of 
positioning system in an indoor environment which is only using trilateration algorithm.

A localization method based on RSSI which is using EKF algorithm and the circulary antenna is proposed in [17]. The EKF implement Position-velocity (PV) model, which assumes that the target is moving at a near-constant velocity. There are 4 reference nodes deployment at $3.5 \mathrm{~m} \mathrm{x} 3 \mathrm{~m}$ area. The smaller area which has not complex propagation characteristics can be used various PLE value for each reference. However, this system is not suitable for the complex indoor environment that has various PLE value from fluctuative RSSI data.

The EKF algorithm is not stable when it applied for the strongly non-linear system as well as RSSI positioning system at an indoor environment. The IEKF is the solution of it which provides more accurate estimates than EKF. There are many kinds of parameters for processing the estimation at the IEKF algorithms such as position which have obtained before using RSSI, TOA, TDOA positioning system, velocity, acceleration or using inertial navigation system from IMU sensors [1], [18]. The IEKF algorithm performs significantly better when it combined with the other algorithm such as particle filter [19].

However combining with the other algorithm as Particle filter and IEKF algorithm which have iteration process in them, it will influence the computation time of the system. In order to overcome this problem, the IEKF algorithm requires the modified algorithm such as adaptive IEKF. The adaptive IEKF has automatically stopped the iterations when there is no change in the next iteration. Another modification research of IEKF is improving the IEKF with only one GN (Gauss-Newton) method iteration which is suitable for a control of the step-length iterations [11].

Combining the cooperative tracking scenario and the IEKF algorithm is also suitable for the tracking system which requires high accuracy and low computation. We extend our previous work at [7], that was proposed cooperative tracking system based on three nearest distance of the anchor node as the initial position estimation. The initial position calculation will be added cluster-based PLE area system for grouping the PLE value from the area which have almost same propagation characteristics. EKF algorithm was also added to improve the position estimation from trilateration method. Due to the IEKF performance is better than EKF algorithm, we propose modified IEKF algorithm which was extended our previous work to achieve better performance in error estimation or accuracy system and the computation time calculation.

\section{B. Trilateration Algorithm}

Trilateration is an algorithm which estimates the position of mobile node based the distances from anchor nodes to the mobile node to be located at three nearest anchor nodes [4][6], [8]. The distance is derived from RSSI measurement between anchor nodes as the reference to a mobile node. There are anchor nodes coordinate $\left(x_{1}, y_{1}\right),\left(x_{2}, y_{2}\right),\left(x_{3}, y_{3}\right)$ which estimate mobile node $\left(x_{\text {est }}, y_{\text {estl }}\right)$ as a central circle by intersecting their three circles, as shown in Fig. 1. The mobile node is assumed placed on the intersection point.

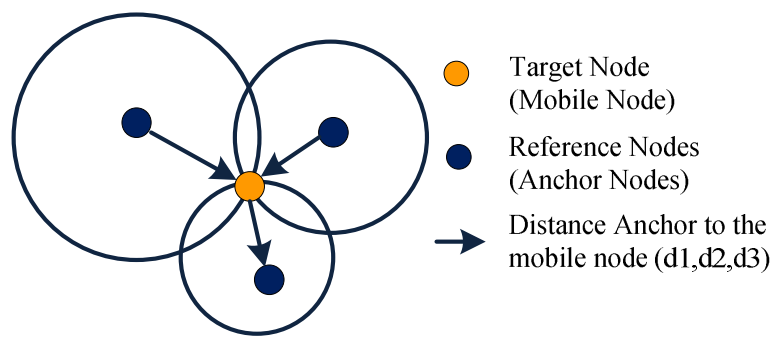

Fig. 1 Trilateration algorithm illustration

The distance from each anchor node to the mobile node will represent as $d_{i}(i=1,2,3 \ldots, n)$. The distance estimation which is obtained from range extension of RSSI measurement is derived by the euclidean distance between the midpoint of each circle and their intersection point as follows:

$$
\left(x_{i}-x_{e s t 1}\right)^{2}-\left(y_{i}-y_{e s t}\right)^{2}=d_{i}^{2}
$$

Each part of Eq. (1) will be subtracted with the other part distance which can be obtained to this following equation using matrix formula based on number anchor node as reference node respectively:

$$
P=\left(A^{T} A\right)^{-1} A^{T} B
$$

Assume, $i=1,2,3 \ldots, n$ are reference nodes with coordinated $\left(x_{i}, y_{i}\right)$ and RSSI data from measurement phase yields estimated distance, $d_{i}$. The mobile nodes will calculate its position $\left(x_{\text {est }}, y_{\text {est }}\right)$ using trilateration or multilateration based its number reference formulas as follows [20]:

$$
P=\left[\begin{array}{l}
x_{e s t} 1 \\
y_{\text {est } 1}
\end{array}\right] \rightarrow A=2\left[\begin{array}{cc}
\left(x_{i=1}-x_{i+1}\right) & \left(y_{i=1}-y_{i+1}\right) \\
\left(x_{i=1}-x_{i+2}\right) & \left(y_{i=1}-y_{i+2}\right) \\
\ldots & \ldots \\
\left(x_{i=1}-x_{n}\right) & \left(y_{i=1}-y_{n}\right)
\end{array}\right]
$$

The B matrix component can be calculated using this following equation:

$$
B=\left[\begin{array}{c}
d_{i+1}^{2}-d_{i=1}^{2}-\left(x_{i+1}^{2}+y_{i+1}^{2}\right)+\left(x_{i=1}^{2}+y_{i=1}^{2}\right) \\
d_{i+2}{ }^{2}-d_{i=1}^{2}-\left(x_{i+2}{ }^{2}+y_{i+2}{ }^{2}\right)+\left(x_{i=1}^{2}+y_{i=1}^{2}\right) \\
\cdots \\
d_{n}{ }^{2}-d_{i=1}^{2}-\left(x_{n}{ }^{2}+y_{n}^{2}\right)+\left(x_{i=1}^{2}+y_{i=1}^{2}\right)
\end{array}\right]
$$

In this system, the estimation output from trilateration was the initial estimation $\left(x_{e s t l}, y_{\text {estl }}\right)$ of the mobile cooperative tracking system from 3 nearest references node. When it is added more than 3 nearest references node, it will be called multilateration algorithm. Due to the weakness of accuracy level of trilateration method which is used varied RSSI data, EKF algorithm is added to improve the estimation output.

\section{Extended Kalman Filter (EKF)}

One of an algorithm which can be applied for non-linear system especially in tracking object is extended Kalman filter (EKF). Indoor positioning based RSSI measurement have varied data that make non-linear characteristic to this system. Due to NLOS propagation effect of indoor environment of this system, EKF algorithm could be used to assist the refining process of estimation position from 
trilateration method. There are 3 types of EKF which are widely used in the tracking system; those are P-model (position), PV-model (position, velocity) and PVA (position, velocity, acceleration) [4]. According to the system that used RSSI as a parameter to determine the position, the suitable type of EKF is P-model.

In P-model, EKF of this system is calculating the position based on the result $\left(x_{t r i}, y_{t r i}\right)$ of trilateration. As shown in Fig. 2, there are 3 phases of EKF algorithm: initialization, prediction and update [1]-[4], [7], [9], [13]-[15], [17]-[21].

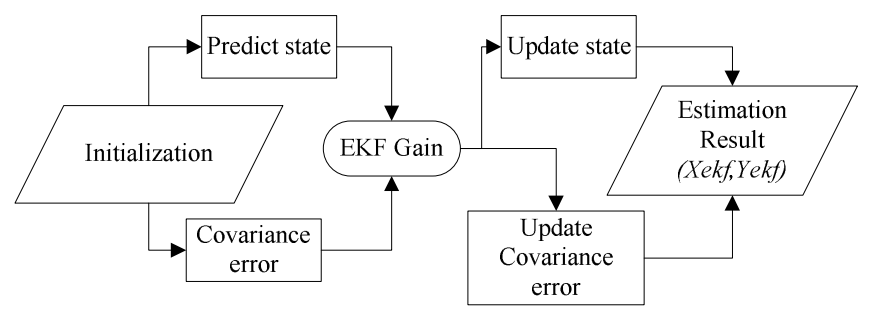

Fig. 2 Diagram block of P-Model EKF algorithm

\section{1) Initialization State}

This step has basically modeled the equation and the parameter which will be processed using EKF algorithm as follows [4]:

$$
x_{k}=f\left(x_{k-1}\right)+W_{k-1}
$$

The result of modeled state based on observation data is derived as:

$$
z_{k}=h\left(x_{k}\right)+v_{k}
$$

$W_{k-1} \sim N\left(0, Q_{k}\right)$ and $v_{k-1} \sim N\left(0, R_{k}\right)$ variable are the noise value which has a normal distribution with zero average value, and its covariance matrix is $Q_{k}$ and $R_{k} . x_{k}$ and $x_{k-1}$ value is the state vector at the $t_{k}$ and $t_{k-1}$ time, while $f$ is the non-linear function which is used for predicting data information based on previous data, as the $h$ function in decrypting the correlation state among $x_{k}$ and $z_{k}$.

\section{2) Predict State}

In this step will be predicted variable that has been declared in the initialization state. Priori state $\hat{x}_{k \mid k-1}$ processed the previous information data from the posteriori state $\hat{x}_{k-1 \mid k-1}$ which was described by the following equation [4]:

$$
\hat{x}_{k \mid k-1}=F \cdot \hat{x}_{k-1 \mid k-1}+B_{k} \cdot u_{k}
$$

$\mathrm{u}_{\mathrm{k}}$ denotes the input system, $\mathrm{F}$ is the transition matrix and $\mathrm{B}_{\mathrm{k}}$ as the input matrix. Matrix covariance calculation can be obtained as follow:

$$
P_{k \mid k-1}=F \cdot P_{k-1 \mid k-1} \cdot F^{T}+Q
$$

The variable of $P_{k \mid k-1}$ and $P_{k-1 \mid k-1}$ are the information state which was related to the previous covariance data $\mathrm{Q}$.

\section{3) Update State}

The updating state will be processed after the predicting data have been done. The output from this state is the expected estimation result. There is an innovation vector $\tilde{Y}_{k}$ from eq.(6) modification as follow [4]:

$$
\tilde{Y}_{k}=z_{k}-h\left(\hat{x}_{k}\right)
$$

The equation of covariance matrix $S_{k}$ based on observation result is derived as:

$$
S_{k}=H_{k} \cdot P_{k \mid k-1} H_{k}^{T}+R_{k}
$$

$H_{k}$ is the Jacobian matrix based on expected measurement result value from $h\left(\hat{x}_{k}\right)$. The posteriori state which is the estimation result from EKF algorithm can be calculated by the following equation:

$$
\hat{x}_{k \mid k}=\hat{x}_{k \mid k-1}+K_{k} \cdot \tilde{Y}_{k}
$$

$\mathrm{K}_{\mathrm{k}}$ is the Kalman gain from the following calculation:

$$
K_{k}=P_{k \mid k-1} \cdot H_{k}^{T} \cdot S_{k}^{-1}
$$

The covariance matrix from the estimation result is using $\mathrm{I}_{\mathrm{n}}$ identity matrix $n \times m$ as follows:

$$
P_{k \mid k}=\left(I_{n}-K_{k} \cdot H_{k}\right) \cdot P_{k \mid k-1}
$$

\section{Iterated Extended Kalman Filter (IEKF)}

The EKF algorithm has better performance in mildly nonlinear functions, but if the observation matrix equation (6) is strongly nonlinear (such as in tracking system), the performance of the EKF deteriorates. In such situations, the iterated EKF (IEKF) tends to provide more accurate estimates than EKF [11], [12], [19], [24].

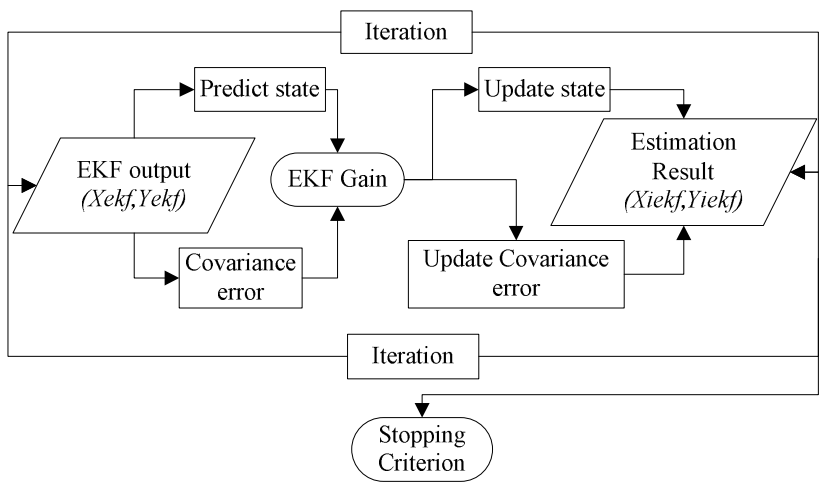

Fig. 4 Diagram block of IEKF algorithm

IEKF algorithm can be improved the estimation output from EKF algorithm. Those improvements are achieved by local iterations or forward processing from the EKF measurement update. Basically, the main process of the IEKF is almost same with EKF algorithm which used initialization, prediction, and updates. However, there are some number iterations which can influence the estimation result as shown in Fig. 3. The iterations should be stopped when there is no significant change in the estimation result.

\section{1) Initialization State}

In initialization state of IEKF, first, should set the iteration $i=0$. After that, the state vector $\left(\mathrm{x}_{\mathrm{k}}\right)$ is replaced by the estimation output of EKF algorithm. In this case, the EKF process is the first iteration of this system. According to the switching of the state vector at every iteration, the equation of state vector of the IEKF can be derived as:

$$
\hat{x}_{k}^{0}=\hat{x}_{k \mid k-1}
$$




\section{2) Predict State}

In this step will calculate the same process as the EKF algorithm in equation (7-8). Furthermore, it needs to be replaced the state with the estimation result from EKF algorithm.

\section{3) Update State}

The update states of IEKF were also obtained from each result of EKF algorithm. The main state is Jacobian matrix $H_{k}$ which is derived as [11]:

$$
H_{k}{ }^{i}=\left.\frac{\partial h_{k}(s)}{\partial s}\right|_{s=\hat{x}_{k}{ }^{i}}
$$

The Kalman gain should be changed by its iteration as follow:

$$
K_{k}^{i}=P_{k \mid k-1 \cdot\left[H_{k}{ }^{i}\right]^{T} \cdot S_{k}}^{-1}
$$

The posteriori state as the estimation result of IEKF algorithm is represented as:

$$
\hat{x}_{k \mid k}^{i+1}=\hat{x}_{k \mid k-1}+K_{k}^{i} \cdot\left(z_{k}-h_{k}\left(\hat{x}_{k}^{i}\right)-H_{k}{ }^{i}\left(\hat{x}_{k \mid k-1}-\hat{x}_{k}^{i}\right)\right)
$$

All process of the IEKF will be repeated with $i=i+1$ until a stopping criterion met $[11,12]$. Stopping criterion is a parameter to determine the maximum number iterations of IEKF algorithms which are the larger iterations can be influenced to the time computation. The stopping criterion is represented as delta. If delta is smaller than the threshold, the iterations process will be stopped and turn the final value of estimation result of IEKF. Otherwise, when the delta larger than the threshold, it will continue the iterations process based on the modeling system from the estimation result of each iteration. The value of delta is derived from this following equation [11]:

$$
\text { delta }_{i}=g\left(x_{k}^{i}-x_{k}^{i-1}\right)
$$

$g$ function is the difference function among the posterior state with the result of posterior state at the previous iteration. The difference function is the calculated based on the Euclidean distance between them. After that, in comparing the value of delta with the threshold is used the average value calculation. the threshold is determined from the first delta $\left(\right.$ delta $\left._{i=1}\right)$ calculation. If the the delta $a_{i+1}$ larger than $\operatorname{delta}_{i=1}$, the iterations process will be continued until

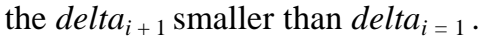

\section{E. Proposed Modified IEKF Algorithm}

The tracking system has a strongly non-linear characteristic, particularly in using RSSI based positioning system. Due to the non-linear problem of this mobile tracking system, there are 4 required estimation processes, as illustrated in Fig. 4. The first estimation is obtained from trilateration method using three nearest distance reference. Combined the trilateration method with the EKF algorithm will be achieved the improvement result. Using EKF algorithm can be decreased the error estimation and linearized the initial estimation result.

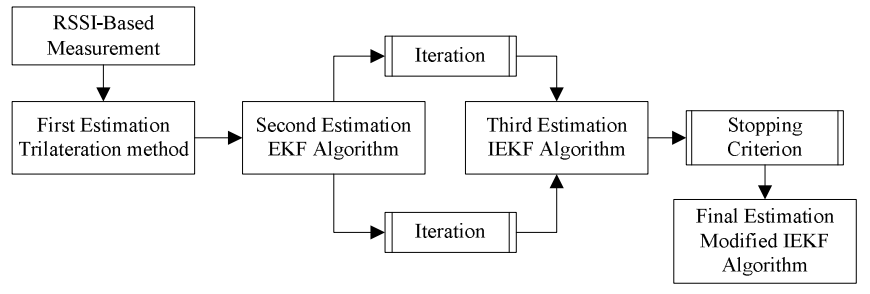

Fig. 4 Block diagram of modified IEKF for mobile cooperative tracking system

The combination of trilateration and EKF algorithm will be processed by 3 steps involves initialization state, update state and predict state as the original EKF algorithm. The initialization variables can be described as follows:

$$
x_{k}=\left[\begin{array}{ll}
x_{t r i} & y_{t r i}
\end{array}\right]
$$

The value of $z_{k}$ is used distance estimation from 3 nearest anchor node that can be described as follows:

$$
z_{k}=\left[\begin{array}{lll}
d_{\text {est } 1} & d_{\text {est } 2} & d_{\text {est } 3}
\end{array}\right]
$$

$\mathrm{d}_{\text {est }(1,2,3)}$ is the distance estimation of the mobile node to the anchor node that was obtained from PLE value eq. (1727). The covariance matrix is obtained from the coordinate estimation of trilateration method that is described in this following matrix:

$$
P_{0}=\left[\begin{array}{ccc}
\sigma^{2} x_{t r i} & 0 & 0 \\
0 & \sigma^{2} y_{t r i} & 0 \\
0 & 0 & 0
\end{array}\right]
$$

The variable that has been declared before in Eq. (19), (21) will be predicted to the next state using the predicted state with this following equation:

$$
\begin{gathered}
x_{k}=\left[\begin{array}{cc}
x_{t r i} & y_{t r i}
\end{array}\right]^{T} * F \rightarrow F=\left[\begin{array}{ccc}
1 & 0 & 0 \\
0 & 1 & 0 \\
0 & 0 & 1
\end{array}\right] \\
P_{k}=F * P_{0} * F^{T}+Q \rightarrow Q=P_{0}
\end{gathered}
$$

$P_{k}$ is information state that related with covariance information data before and $Q$ is matrix covariance. The update state from eq.(22-23) will be multiplied by the Kalman gain $\left(\mathrm{K}_{\mathrm{k}}\right)$. The value of $\mathrm{K}_{\mathrm{k}}$ is derived as:

$$
K_{k}=P_{k} \cdot H_{k}^{T} \cdot S_{k}^{-1}
$$

The declaration value of $\mathrm{K}_{\mathrm{k}}$ is influenced by jacobian matrix $\mathrm{H}_{\mathrm{k}}$ that is expected the result of estimation data to the real data and also influence from updating noise or covariance matrix $\left(P_{k}, S_{k}\right)$. The update state can be obtained using this following equation:

$$
\begin{gathered}
H_{k}=\left[\begin{array}{lll}
\frac{x_{\text {tri } 1}-x_{\text {anchor } 1}}{\text { dist }_{1}} & \frac{y_{\text {tri } 1}-y_{\text {anchor } 1}}{\text { dist }_{1}} & 0 \\
\frac{x_{\text {tri } 2}-x_{\text {anchor } 2}}{\text { dist }_{2}} & \frac{y_{\text {tri } 2}-y_{\text {anchor } 2}}{\text { dist }_{2}} & 0 \\
\frac{x_{\text {tri } 3}-x_{\text {anchor } 3}}{\text { dist }_{3}} & \frac{y_{\text {tri } 3}-y_{\text {anchor } 3}}{\text { dist }_{3}} & 0
\end{array}\right] \\
\operatorname{dist}_{k(1,2,3)}=\sqrt{\left(x_{\text {tri }(k)}-x_{\text {anchor }(k)}\right)^{2}+\left(y_{\text {tri }(k)}-y_{\text {anchor }(k)}\right)^{2}}
\end{gathered}
$$


Covariance matrix value $S_{k}$ is calculated by a combination of covariance matrix $\mathrm{P}_{\mathrm{k}}$ from update state which is added with a noise variance of distance estimation $\mathrm{R}_{\mathrm{k}}$ derived as:

$S_{k}=H_{k} \cdot P_{k} \cdot H_{k}^{T}+R_{k} \rightarrow R_{k}=\operatorname{diag}\left(\sigma^{2} d_{e s t 1} \quad \sigma^{2} d_{e s t 2} \quad \sigma^{2} d_{e s t 3}\right)$

According to the update state, the value of $\mathrm{P}_{0}$ that has been declared before is derived as:

$$
P_{0}=\left(P_{k}-K_{k} * H_{k}\right) * P_{k}
$$

It also influences the observation matrix that can be calculated as follows:

$$
y_{k}=z_{k}-h_{k} \rightarrow h_{k}=\left[\begin{array}{lll}
\text { dist }_{1} & \text { dist }_{2} & \text { dist }_{3}
\end{array}\right]
$$

The posterior state which is estimation result from EKF algorithm will result in coordinate output $\left(\mathrm{x}_{\mathrm{EKF}}, \mathrm{y}_{\mathrm{EKF}}\right)$ :

$$
x_{k}=x_{k}+K_{k} \cdot y_{k}
$$

The estimation result from trilateration and EKF algorithm still achieved high error estimation result. Furthermore, it can be reduced using IEKF algorithm which is designed for the strongly non-linear characteristic. In IEKF algorithm, the output from EKF algorithm is assumed as first iteration result from IEKF. The state vector of IEKF algorithm $\left(\mathrm{x}_{\mathrm{k}}\right)$ is replaced by the estimation output of EKF algorithm which is set at the initial iteration $i=0$. According to the switching of the state vector at every iteration, the equation of state vector of the IEKF can be derived as:

$$
x_{k^{i}}=\left[\begin{array}{ll}
x_{e k f_{i}} & y_{e k f_{i}}
\end{array}\right]
$$

It is also followed by observation matrix $\left(\mathrm{z}_{\mathrm{k}}\right)$ that was measurement data from distance estimation of EKF algorithm. The distance estimation can be calculated using Euclidean distance as equation (26) between the coordinate estimation of EKF to the three nearest anchor node coordinate. The observation matrix will be updated based on the iteration process which is described by the following equation:

$$
z_{k} i=\left[\begin{array}{lll}
d_{1 e k f_{i}} & d_{2 e k f_{i}} & d_{3 e k f_{i}}
\end{array}\right]
$$

The covariance matrix $P_{0}$ is obtained from the coordinate estimation of each iteration estimation result. The predicted state of IEKF algorithm should be replaced the state with EKF algorithm result. The main state from the update state process is Jacobian matrix $\mathrm{H}_{\mathrm{k}}$ which is derived as:

$$
H_{k}^{i}=\left[\begin{array}{lll}
\frac{x_{1 e k f_{i}}-x_{\text {anchor } 1}}{d 1_{e k f_{i}}} & \frac{y_{1 e k f_{i}}-y_{\text {anchor } 1}}{d 1_{\text {akf }}} & 0 \\
\frac{x_{2 e k f_{i}}-x_{\text {anchor } 2}}{d 2_{e k f_{i}}} & \frac{y_{2 e k f_{i}}-y_{\text {anchor } 2}}{d 2_{e k f_{i}}} & 0 \\
\frac{x_{3 e k f_{i}}-x_{\text {anchor } 3}}{d 3_{e k f_{i}}} & \frac{y_{3 e k f_{i}}-y_{\text {anchor } 3}}{d 3_{e k f_{i}}} & 0
\end{array}\right]
$$

Covariance matrix value $S_{k}, P_{k}$ and noise variance of distance estimation $R_{k}$ are calculated using equation (27-30) which is also replaced some parameter output of trilateration with EKF algorithm.

Using IEKF algorithm can be decreased the error estimation. However, due to the strongly non-linear characteristic data make the reduction of the estimation at each iteration will be different. This condition required addition method to modify the IEKF algorithm based each iteration result.
TABLE I

EXAMPLE DATA OF IEKF ESTIMATION

\begin{tabular}{|c|c|c|c|c|c|}
\hline \multicolumn{5}{|c|}{ MSE (meters) } \\
\hline No. & TRI & IEKF-1 & IEKF-2 & IEKF-3 & IEKF-4 \\
\hline 1. & 3.29 & 0.48 & 0.54 & 0.61 & 0.67 \\
\hline 2. & 4.78 & 2.71 & 2.56 & 2.46 & 2.38 \\
\hline 3. & 2.31 & 1.47 & 1.10 & 1.19 & 1.41 \\
\hline 4. & 2.77 & 1.23 & 1.39 & 1.44 & 1.45 \\
\hline 5. & 4.25 & 1.73 & 1.56 & 1.42 & 1.31 \\
\hline
\end{tabular}

As shown in Table 1, those are the example data of the estimation result from the mobile cooperative tracking scenario. There are 4 iterations process of IEKF at mobile cooperative tracking scenario. The maximum number of iterations process is calculated based on stopping criterion at equation (18). It shows that data 2 and 5 is decreasing effectively at each iteration, while data 1,3 and 4 are not constantly decreasing. It proves that IEKF algorithm is unstable for reducing the error estimation at each iteration. There are many factors that can cause its situation. One of them is modeling system in covariance noise. Learning process of each data to make specific modeling system is

\begin{tabular}{|c|c|}
\hline & fied IEKF Algorithm \\
\hline & $\mathrm{R} n=1$ to $\mathrm{N}$ \\
\hline 2: & Initialization \\
\hline & $\mathrm{X}_{\text {IEKF }}=\left[\mathrm{x}_{\text {IEKF1 }} \mathrm{x}_{\text {IEKF2 } 2} \mathrm{x}_{\text {IEKF3 }} \mathrm{x}_{\text {IEKF4 }}\right]$ \\
\hline & $Y_{\text {IEKF }}=\left[y_{\text {IEKF1 } 1} \mathrm{y}_{\text {IEKF2 }} \mathrm{y}_{\text {IEKF3 } 3} \mathrm{y}_{\text {IEKF } 4}\right]$ \\
\hline 3: & Hitung \\
\hline & $\mathrm{X}_{\text {average_IEKF }}=\operatorname{mean}\left(\mathrm{X}_{\mathrm{IEKF}}\right)$ \\
\hline & $\mathrm{Y}_{\text {average_IEKF }}=\operatorname{mean}\left(\mathrm{Y}_{\mathrm{IEKF}}\right)$ \\
\hline 4: & IF $X_{\text {average_EKF }}>X_{\text {real }}$ \\
\hline & $X_{\text {new_EKF }}=\min \left(X_{\text {IEKF }}\right)$ \\
\hline 5: & ELSE IF $X_{\text {average_EKF }}<X_{\text {real }}$ \\
\hline & $X_{\text {new_EKF }}=\max \left(X_{\text {IEKF }}\right)$ \\
\hline 6: & ELSE IF $Y_{\text {average_IEKF }}>Y_{\text {real }}$ \\
\hline & $Y_{\text {new_IEKF }}=\min \left(Y_{\text {IEKF }}\right)$ \\
\hline & ELSE IF $Y_{\text {average_EKF }}<Y_{\text {real }}$ \\
\hline & $Y_{\text {new_IEKF }}=\max \left(Y_{\text {IEKF }}\right)$ \\
\hline & $D$ for $\mathrm{N}$ \\
\hline
\end{tabular}
very complicated and need a long time. In our proposed modified IEKF, we will utilize the existing data to take selection process with an average value approach. The modified IEKF algorithm of the mobile cooperative tracking system is described as :

According to the modified IEKF algorithm, it will be compared the coordinate result from each IEKF iteration with the real coordinate route of the object. It is because, in this cooperative tracking system, we assume the initial point route and final point route of the object. Using this algorithm, the reduction will be stable although each iteration is not constantly reduced. The result of the modified IEKF will be presented at the next section of experiment and discussion.

\section{RESULTS AND DISCUSSION}

In this section will be described the design system, scenario, and procedure of this tracking system. There are two main steps of this system which are RSSI measurement phase and mobile cooperative tracking simulation. After that, the performance of this system will be analyzed based on error estimation and time computation. 


\section{A. Measurement Phase}

In this phase, RSSI data are measured from Xbee-Pro (S2) module which is implemented at communication process among nodes. Anchor node as the node reference or transmitter was transmitted RSSI data to the mobile node as the target node or the receiver. RSSI measurement result will be used for determining distance estimation among anchor node and the mobile node. However, calculating the distance estimation from RSSI data need propagation characteristic which can be obtained from PLE (path loss exponent) value and deviation standard. There are three measurement scenarios that were conducted at this system. Two scenarios were used LOS condition for determining the PLE value and deviation standard of this system. One scenario was occurred in NLOS condition for obtaining the RSSI data based on the assumption route of the mobile node to the nearest anchor. The measurement scenarios are held on the $3^{\text {rd }}$ floor of applied postgraduate EEPIS building based on its indoor environment.

\section{1) First Scenario}

Anchor nodes position is placed at $2.4 \mathrm{~m}$ height, while the mobile node is placed at $0.9 \mathrm{~m}$ height. First scenario measurement is determining the effect of distance modification to the RSSI data. It will be measured reference data at $1 \mathrm{~m}$ among anchor node and the mobile node. After that, anchor node stayed in one position, and mobile node moved every $2 \mathrm{~m}$ distance in LOS (line of sight) condition, as shown in Fig. 5. Every $2 \mathrm{~m}$ movement, there are 10 RSSI data which will be measured.

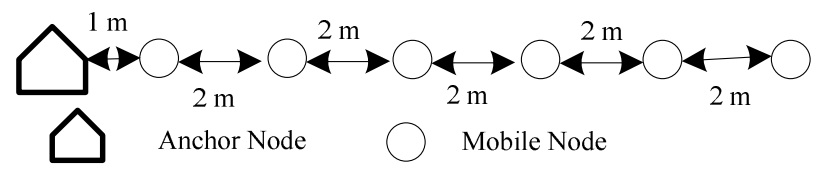

Fig. 5 First measurement scenario

\section{2) Second Scenario}

Like the first scenario, the height position of anchor node and mobile node still same at $2.4 \mathrm{~m}$ and $0.9 \mathrm{~m}$. The second scenario is using same distance and different position among anchor node and the mobile node. This scenario is used to get deviation standard based on noise variance which is resulted from the difference value of RSSI data inter-node based its modification position, as shown in Fig. 6. There are 3 main positions to measure the deviation standard. Those are the vertical, horizontal and tilted position. In that position can be applied randomly based on tilt, but it should stay in $2 \mathrm{~m}$ distance among anchor node and the mobile node.

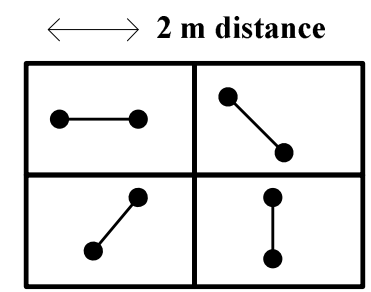

Fig. 6 Second measurement scenario
According to the RSSI measurement data from that scenario, it can be used to calculate PLE value. There are two kinds of PLE values calculation which were used in this system. Those are PLE value based on measurement approach and linear regression approach. The PLE value based on measurement calculation is using RSSI data to estimate the distance that is given as [25]:

$$
R S S I=-\left(10 n \log _{10}(d)+A\right)
$$

$n$ is the PLE value that is influenced by $d$ distance from transmitting node and $A$ the RSSI at 1-meter distance as reference data. From Eq. (34) PLE value measurement based on a real condition is derived as [25]:

$$
n=\frac{A+R S S I}{-\left(10 \log _{10} d\right)}
$$

PLE value based on linear regression is calculated from modeling slope of the line to the fluctuating RSSI data that have gradient value.

$$
y=a+b x
$$

From Eq. (36) $b$ is the coefficient of slope line, and this parameter can be substituted to this equation so that each value is derived as [26], [27]:

$$
\begin{gathered}
y=P L(d) \rightarrow P L(d)=P L+10 n \log _{10}(d) \\
a=P L-10 n \log _{10}(d) \rightarrow P L=E I R P-R S S I \\
b=10 n \\
x=\log (d)
\end{gathered}
$$

$P L$ is the path loss value in $\mathrm{dB}$ that was calculated from the difference value between EIRP and RSSI. EIRP (Effective Isotropic Radiated Power) is the value of transmitting power $(\mathrm{dB})$ plus transmitter antenna gain $(\mathrm{dB})$ and receiver antenna gain $(\mathrm{dB})$. The value of $a$ and $b$ using linear regression equation can be calculated using this equation [14]:

$$
\begin{gathered}
b=\frac{N \sum x_{i} y_{i}-\left(\sum x_{i}\right)\left(\sum y_{i}\right)}{N \sum x_{i}^{2}-\left(\sum x_{i}\right)^{2}} \\
a=\frac{\sum y_{i}}{N}-b \frac{\sum x_{i}}{N}
\end{gathered}
$$

$N$ is the number of RSSI data that was got from the measurement. The value of PLE, $n$ can be obtained using this equation [14]:

$$
n=\frac{b}{10}
$$

The result of PLE value based on measurement calculation eq. (35) was in the range 0.89 to 1.76 in LOS condition and 2.1 to 5.09 in the NLOS condition. While the range value of PLE based on linear regression calculation eq. (36-43) was 0.96 to 1.86 in LOS condition and 2.14 to 4.98 in NLOS condition.

\section{3) Third Scenario}

After determining the PLE value, distance estimation based on RSSI data can be obtained. RSSI data measurement which is used to obtained will be obtained from measurement phase from assumption route of the scenario, as shown in Fig. 7. There are 53 measurement point of the mobile node in height $0.9 \mathrm{~m}$ with $2 \mathrm{~m}$ separation distance. Anchor node is deployed at the wall of the building in height 
$2.4 \mathrm{~m}$. There are 18 anchor nodes which are deployed with 7 $\mathrm{m}$ separations. The measurement process starts from the first measurement point of the mobile node to the nearest anchor node with $10 \mathrm{~m}$ maximum distance. It means when the mobile node move to the next point route and the distance is in the range $10 \mathrm{~m}$, the measurement process should be stopped and will be measured to the other anchor nodes. This process will be repeated for the every anchor node to the nearest point route of the mobile node.

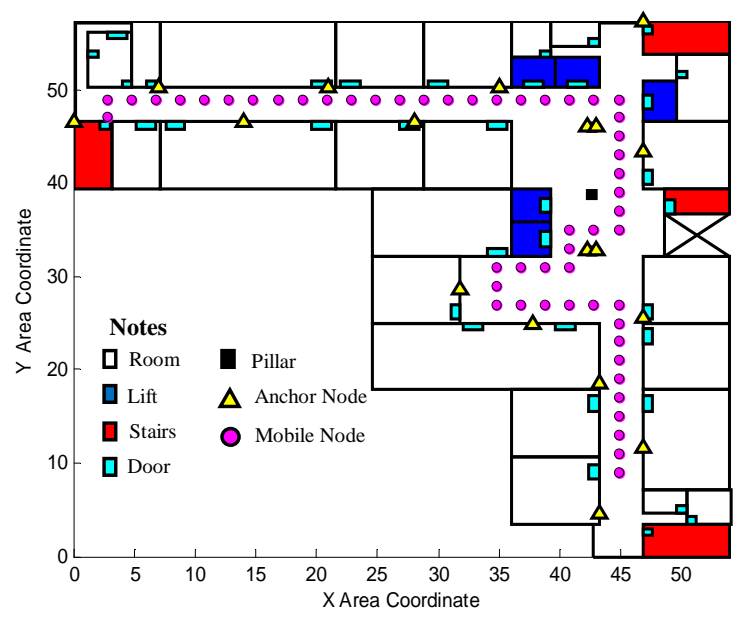

Fig. 7 Topology system of assumption route scenario

After taking RSSI measurement based on assumption route scenario, the distance estimation can be obtained using this equation:

$$
d=10^{-\left(\frac{R S S I+A}{10 n}\right)}
$$

$\mathrm{d}$ is the distance estimation, RSSI data that was obtained from measurement process for every anchor to the mobile node. A is the RSSI data measurement for each anchor at 1 $\mathrm{m}$ distance, while $\mathrm{n}$ is the PLE value which was calculated before in equation (35-43).

\section{B. Mobile Cooperative Tracking Simulation}

After determining the distance estimation based on RSSI measurement phase, it will take the mobile cooperative tracking simulation. In this paper, we simulate a realistic scenario which is implemented in the indoor environment of the $3^{\text {rd }}$ floor of EEPIS postgraduate building. The simulation process is conducted by MATLAB software. The relevant parameter simulation of this system is listed in Table 2.

TABLE II

SIMULATION PARAMETER

\begin{tabular}{|l|l|l|}
\hline Parameters & Value & Remarks \\
\hline Operating Frequency & $2.4 \mathrm{GHz}$ & \multirow{2}{*}{ Xbee-PRO (S2) module } \\
\cline { 1 - 2 } Transmit power (EIRP) & $17 \mathrm{~dB}$ & \\
\hline Receiver sensitivity & $-102 \mathrm{dBm}$ & $\begin{array}{l}\text { Anchor node } \\
\text { deployment }\end{array}$ \\
\cline { 1 - 2 } $\begin{array}{l}\text { Distance between anchor } \\
\text { node }\end{array}$ & $7 \mathrm{~m}$ & $\begin{array}{l}\text { Mobile cooperative } \\
\text { tracking scenario }\end{array}$ \\
\cline { 1 - 2 } $\begin{array}{l}\text { Maximum range } \\
\text { extension coverage }\end{array}$ & $7-10 \mathrm{~m}$ & $\begin{array}{l}\text { Scenario of } \\
\text { mobile node movement }\end{array}$ \\
\hline $\begin{array}{l}\text { Calculating estimation of } \\
\text { position }\end{array}$ & $2 \mathrm{~m} /$ \\
position & 4 & Implemented at IEKF \\
Maximum Iteration & 4 & \\
\hline
\end{tabular}

The realistic scenario is arranged from the RSSI measurement data result such as, distance, anchor node coordinate deployment, mobile node route and PLE value which was used clustering system. PLE value with their area was stored in a database system. The anchor nodes for the specific position will be distributed the PLE value involve the RSSI data to the mobile node. The Xbee-Pro module with S2 type using $2.4 \mathrm{GHz}$ frequency as well as wifi technology, have a little impact from wifi signal which has different channel [28]. However, the number of Xbee deployment can be influenced to the other Xbee, as well as interference problem. Due to this paper limitation, we assume there is not interference effect to this system by arranging to the different channel for 4 nodes such as three anchor nodes and one mobile node based on this mobile cooperative tracking scenario.

Mobile cooperative tracking simulation start from determining position estimation of the mobile node using trilateration method. Each point route of the mobile node was served by 3 nearest anchor node with maximum range 7-10 $\mathrm{m}$. The maximum range was determined from stability condition of RSSI data which was obtained from the measurement process. When the mobile node moves to the next point route, the mobile node will be served by another anchor node which was nearest to the mobile node. The illustration of mobile cooperative tracking scenario is depicted in Fig. 8. When a mobile node moves to the route 1, anchor node 1, 2, 5 will send RSSI to the mobile node. While, when the mobile node moves to the route 2 anchor node 5 will be replaced with anchor node 6 . In route 2 , the mobile node will be received RSSI data from anchor node 1 , 2 and 6. This process is continued until the all of the simulation route that has been declared. According to that scenario, the distance estimation which will be processed in trilateration method should be adjusted with the anchor node serving to every route of the mobile node.

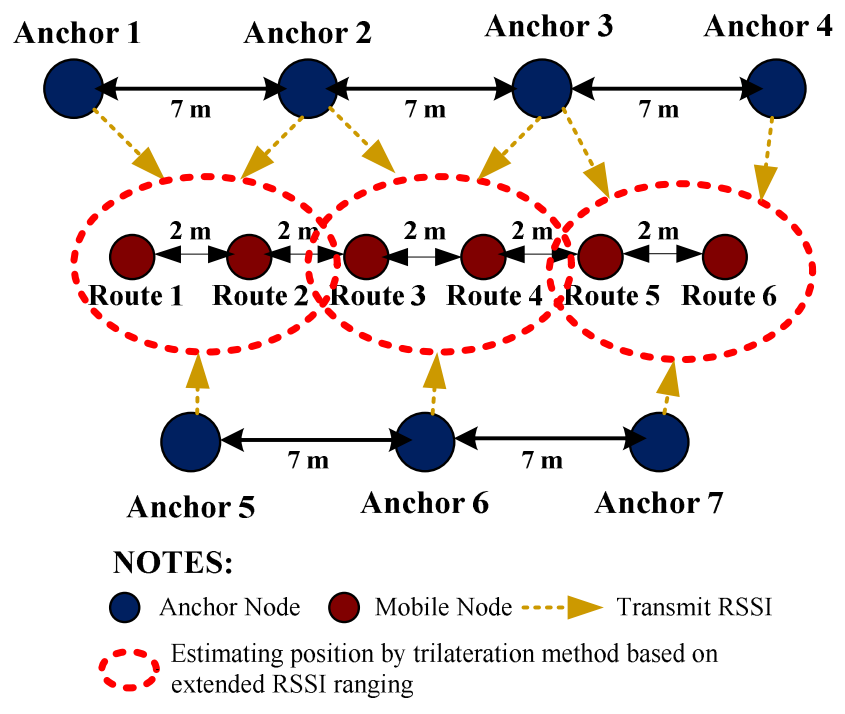

Fig. 8 Mobile cooperative tracking scenario

There are 2 results of distance estimation which will be calculated in trilateration process. Those are distance estimation using PLE measurement value and PLE linear regression value. The results show in Fig. 9 that distance 
estimation using PLE measurement value is near to the linear line indicator than using PLE linear regression value. The linear line is the indicator of the real distance. It proves that distance estimation using PLE measurement value have lower error estimation than using PLE linear regression value. The distance estimation value will be influenced by the position estimation result which will be discussed in the next section.

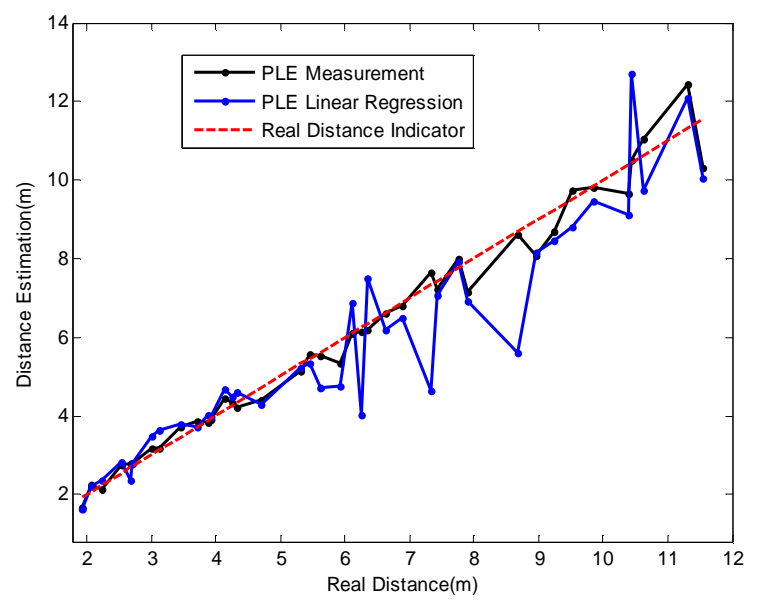

Fig. 9 Distance estimation results based on PLE value

After determining the initial estimation using trilateration method, the next process is obtaining the second estimation using EKF. EKF will be processed the estimation result using trilateration method in each maximum range $(7-10 \mathrm{~m})$ due to the different noise characteristic in each range. EKF algorithm is required to linearize the trilateration output and shows the passing route estimation of the mobile node. This EKF algorithm will be repeated as the IEKF algorithm to reduce the error estimation from EKF algorithm. The output of EKF algorithm is the input of the IEKF algorithm which will be repeated to the next iterations. The maximum number iterations of IEKF is obtained from stopping criterion calculation. There are 4 iterations process of IEKF algorithm to this system. It proves from the example result data of IEKF algorithm which are listed in Table 3.

TABLE III STOPPING CRITERION RESULT

\begin{tabular}{|c|c|c|c|}
\hline & $\left(X_{k}^{2}-X_{k}^{I}\right)$ & $\left(X_{k}^{3}-X_{k}^{2}\right)$ & $\left(X_{k}^{4}-X_{k}^{3}\right)$ \\
\hline & 0.25 & 0.24 & 0.23 \\
\hline & 1.01 & 0.84 & 0.78 \\
\hline & 0.46 & 0.41 & 0.37 \\
\hline & 0.51 & 0.48 & 0.46 \\
\hline & 0.56 & 0.39 & 0.37 \\
\hline Average & 0.57 & 0.47 & 0.44 \\
\hline
\end{tabular}

As shown in Table 3, there are $\left(X_{k}^{2}-X_{k}^{l}\right),\left(X_{k}^{3}-X_{k}^{2}\right)$, and $\left(X_{k}^{4}-X_{k}^{3}\right)$ which are the Euclidean distance between the coordinate result of IEKF from iteration 1 up to iteration 4. From those data will be calculated each average of it. Using the average value can be determined the delta which is used $g$ function as the difference function. The results show that delta $_{1}$ is larger than delta $a_{2}$. It means that iterations process should be stopped. When iterations are continued in those data, there is no significant modification in those data. However, there are some data which are required more than 4 iterations. Due to the iterations process take a long time computation, so in this system will be used 4 maximum iteration process. The performance result of each algorithm estimation of mobile cooperative tracking system will be analyzed using MSE (mean square error) which was represented as:

$$
M S E=\sqrt{\left(x_{\text {real }}-x_{\text {estimation }}\right)^{2}+\left(y_{\text {real }}-y_{\text {estimation }}\right)^{2}}
$$

MSE of this system adopted error distance based on Euclidean distance calculation among real coordinate $\left(X_{\text {real }}\right.$, $\left.Y_{\text {real }}\right)$ and estimation coordinate $\left(X_{\text {estimation, }}, Y_{\text {estimation }}\right)$ from each algorithm.

\section{Performance Analysis}

In this section will be discussed the performance analysis of this system. There are three performances which were evaluated in this system. those are MSE (mean square error) accuracy percentage and time computation. Both of them are comparing with each algorithm which are trilateration, EKF, IEKF and modified IEKF. Time computation is important manner when this system will be implemented in the real scenario. From the time computation result, it can know the correlation among error estimation with the computation time in calculating the position.

\section{1) MSE Performance Analysis}

Regarding the result of position estimation result, we will analyze the error position of each algorithm. The error position of this system can be analyzed in overall data using cumulative distribution function (CDF) as well, as shown in Fig. 10 and Fig.11. This graph is used to know the smallest MSE value based on the cumulative probability for each method.

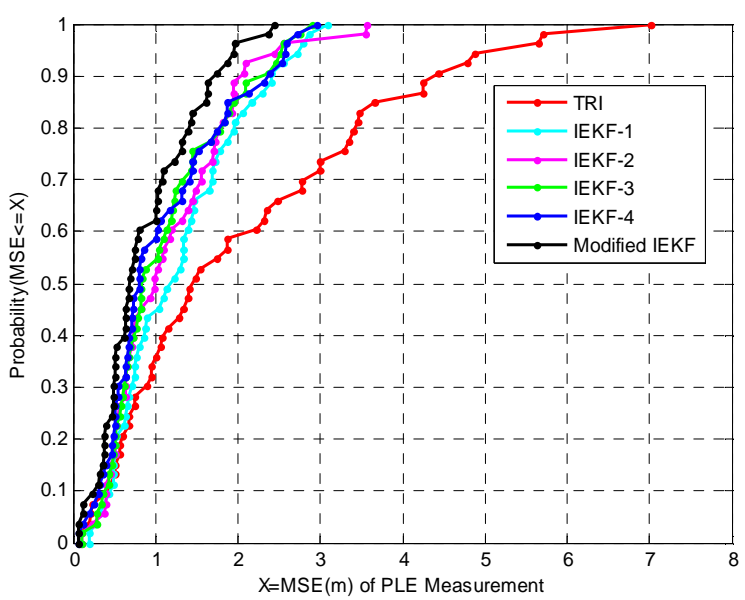

Fig. $10 \mathrm{CDF}$ graph of error position estimation (MSE) using PLE measurement

There are two kinds of PLE value which will influence the estimation result of this system. Those are PLE linear regression value and PLE measurement value. Using PLE measurement value, the best performance is shown by modified IEKF algorithm. It has error estimation range 
between $0.065 \mathrm{~m}$ to $2.4 \mathrm{~m}$. While using IEKF algorithm at the last iteration (IEKF-4), it has almost same error estimation range which are between $0.066 \mathrm{~m}$ to the $2.95 \mathrm{~m}$. It is very different with the initial iteration of IEKF result which has error estimation range up to $3.57 \mathrm{~m}$.

This condition is also followed by using PLE linear regression value which was achieved by modified IEKF algorithm in Fig. 11. However, the estimation error range using PLE linear regression value is larger than using PLE measurement value that is in the range of $0.22 \mathrm{~m}$ to the 2.75 m. Moreover, implemented IEKF algorithm using PLE linear regression value is also got the error reduction at each iteration. At the first iteration to the third iteration of IEKF the error estimation range is $0.28 \mathrm{~m}$ to the $6.83 \mathrm{~m}$, while in the last iteration the error estimation has improvement from $0.27 \mathrm{~m}$ to the $3.54 \mathrm{~m}$.

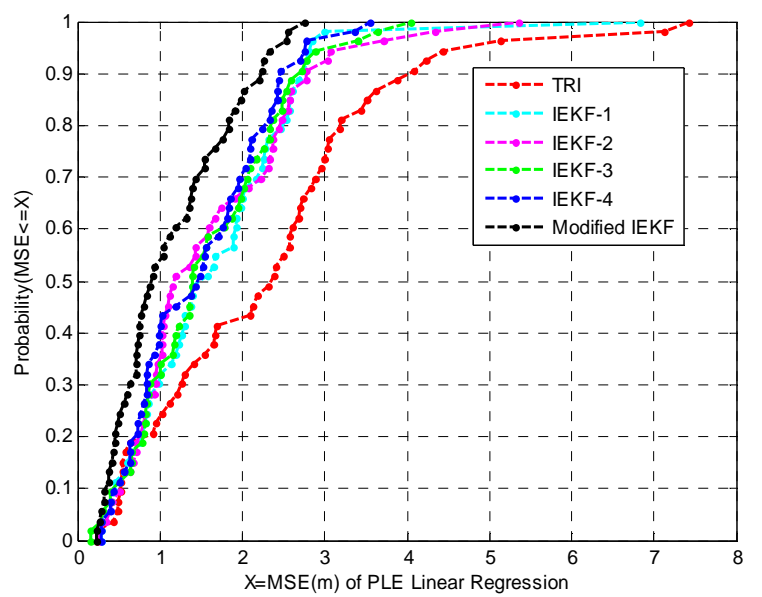

Fig. 11 CDF graph of error position estimation (MSE) using PLE linear regression

According to the data distribution using CDF, MSE average from 53 points route can be calculated as the MSE system which is listed in Table 4. The MSE system comparison shows that modified IEKF algorithm is the better algorithm which has lowest error estimation. Comparing the kind of PLE value which was used in this system, PLE measurement value has better performance than PLE linear regression.

TABLE IV

MSE SYSTEM OF MOBILE COOPERATIVE TRACKING SYSTEM

\begin{tabular}{|c|c|c|}
\cline { 2 - 3 } \multicolumn{1}{c|}{} & $\begin{array}{c}\text { PLE } \\
\text { Measurement }\end{array}$ & $\begin{array}{c}\text { PLE Linear } \\
\text { Regression }\end{array}$ \\
\hline TRI & $2.09 \mathrm{~m}$ & $2.33 \mathrm{~m}$ \\
\hline IEKF-1 & $1.30 \mathrm{~m}$ & $1.67 \mathrm{~m}$ \\
\hline IEKF-2 & $1.19 \mathrm{~m}$ & $1.62 \mathrm{~m}$ \\
\hline IEKF-3 & $1.13 \mathrm{~m}$ & $1.58 \mathrm{~m}$ \\
\hline IEKF-4 & $1.10 \mathrm{~m}$ & $1.49 \mathrm{~m}$ \\
\hline Modified IEKF & $0.88 \mathrm{~m}$ & $1.27 \mathrm{~m}$ \\
\hline
\end{tabular}

Using PLE measurement have $0.88 \mathrm{~m}$ MSE system which was lower than $1 \mathrm{~m}$. While using PLE linear regression was got $1.27 \mathrm{~m}$ which was larger than $1 \mathrm{~m}$. It proves that using PLE measurement value is better that PLE linear regression. This condition can be occurred due to the result of distance estimation. If the distance estimation has higher error estimation, it will influence to the next process. Moreover using PLE measurement value can be described the real environment which was influenced by propagation characteristic.

According to the result of modified IEKF algorithm which has the lowest error, it can be used to the show the passed through the mobile node. In this simulation, it was illustrated the real sketch of the building. It also shows the result of first estimation using trilateration which has linearized using modified IEKF and shows the route estimation from the mobile node, as illustrated in Fig. 12 and Fig. 13.

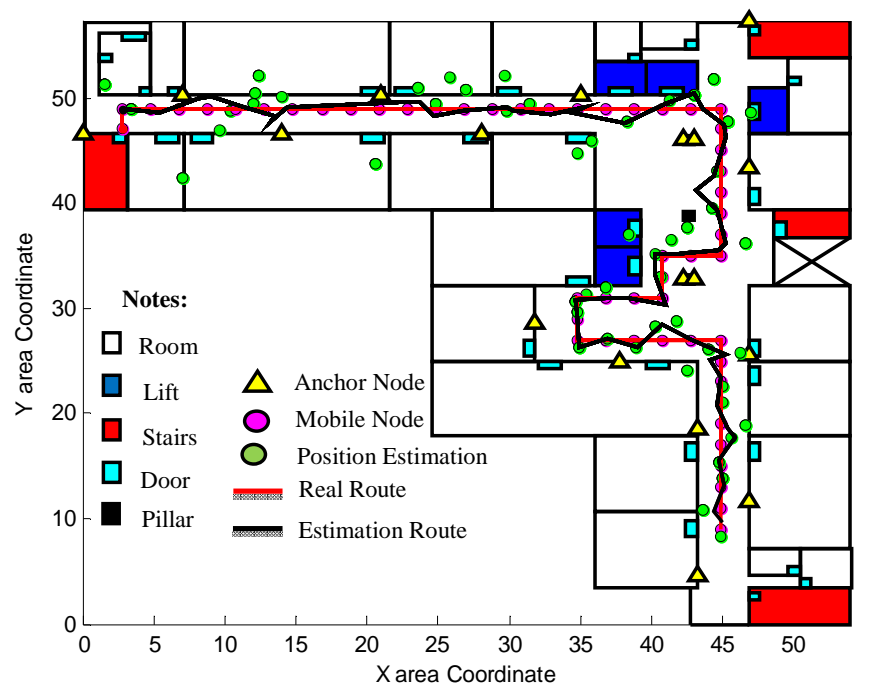

Fig. 12 Route estimation result from modified IEKF algorithm using PLE measurement value

Due to the distribution, MSE data using CDF graphic and MSE system comparison system shows that using PLE measurement have better performance than PLE linear regression. It was influenced to the route estimation of the mobile node. The simulations show that using PLE measurement value is near to the real route. It is in opposite way with the result of using PLE linear regression value in Fig. 13, which is far away to the real route.

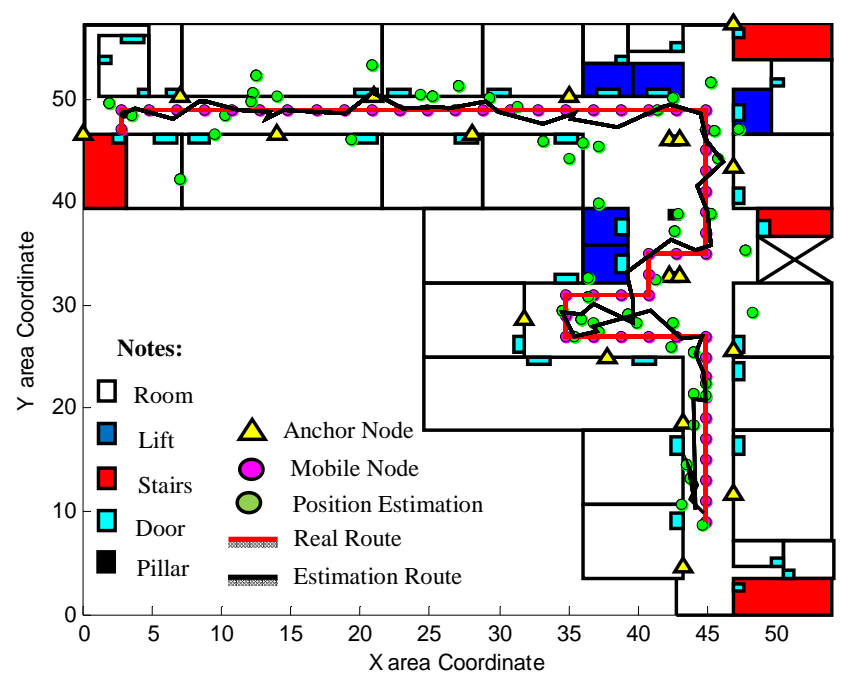

Fig. 13 Route estimation result from modified IEKF algorithm using PLE measurement value 


\section{2) Accuracy Performance Analysis}

The error estimation performance will be influenced by the accuracy level from each algorithm. It is proved from the accuracy level of each algorithm which is shown by percentage level at Fig. 14. The accuracy percentage will be compared for each algorithm implementation from trilateration, IEKF, and modified IEKF. After that, the comparison also followed by the kind of PLE value. The highest accuracy percentage level is achieved by modified IEKF algorithm at $88.11 \%$ using PLE measurement value. This condition also occurs in using PLE linear regression value which has $83.14 \%$ accuracy level. However, adding the conventional IEKF algorithm have the capability for increasing the accuracy level in the range of $1 \%$ to the $7.32 \%$ at each iteration. The improvement accuracy level of conventional IEKF for each iteration is not too high which is caused by the saturation level from stopping criterion IEKF. When it is added more iterations number, the improvement will be same, or there is not modification accuracy level from the previous level.

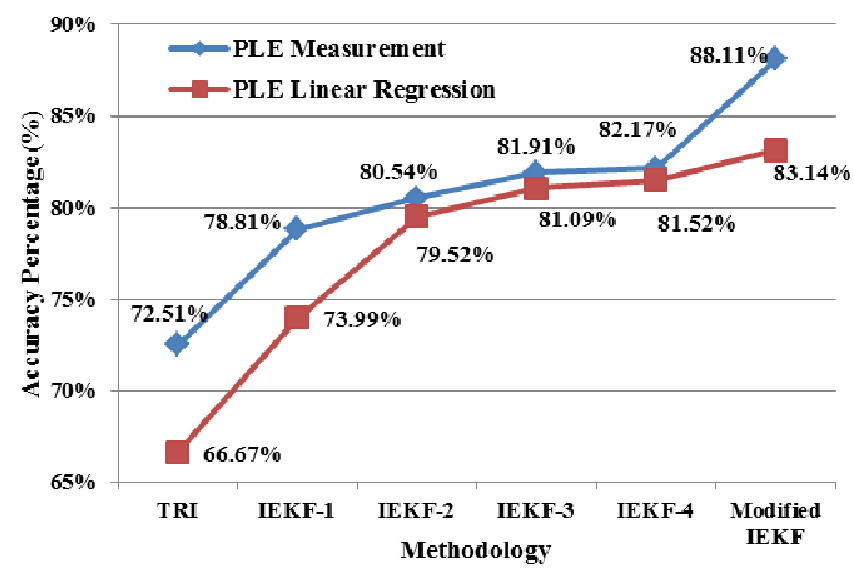

Fig. 14 Accuracy percentage result graph

If the result is compared according to the kind of PLE value, PLE measurement value has higher accuracy level at the all algorithm application than PLE linear regression value. It is because PLE measurement area can be described the real condition of building environment which was obtained from RSSI based measurement. At the same time when the accuracy levels are compared to the MSE performance analysis, it will have some correlations. The higher MSE performance will be received lower accuracy level, and in otherwise the lower MSE performance will be received higher accuracy level.

\section{3) Computation Time Performance Analysis}

In this section will be discussed the time computation of this system for determining the position estimation. Adding IEKF algorithm to this system can influence the computation time performance especially for each iteration. Calculating the computation time of this system can be influenced by the tools and software which was used. Matlab software type and the laptop specification are listed in Table 5.

Comparing the computation time of each algorithm from this system, trilateration has lowest time computation at 0.36 seconds to calculate 53 point route estimations. Using a combination of EKF with the trilateration as shown in IEKF1 data require 0.98 seconds which is larger than only using trilateration method. The increasing computation time of this system will be continued at each iteration. Using IEKF in 4 iterations process take 3.18 seconds which is 3 times larger than using EKF algorithm. Although the iterations process of IEKF can reduce the error estimation effectively. However it take longer computation time to calculate the position estimation, as shown in Fig. 15.

TABLE V

TOOLS SPECIFICATION OF THIS SYSTEM

\begin{tabular}{|l|l|}
\hline Tools & Remarks \\
\hline Laptop & Toshiba Satelite Core i7 M840 \\
\hline Matlab & Type 7.11.0 (R2010b) \\
\hline
\end{tabular}

In this system, we designed of IEKF algorithm without required many iterations process which only takes 4 iteration process. The modified IEKF that used 4 iterations process has computation time at 3.55 seconds. It shows that although modified IEKF has been applied in this system, the computation time of them is almost same to the IEKF-4 algorithm. The kind of PLE value is not too influence the computation time due to PLE value is only variable declaration which does not require long computation time.

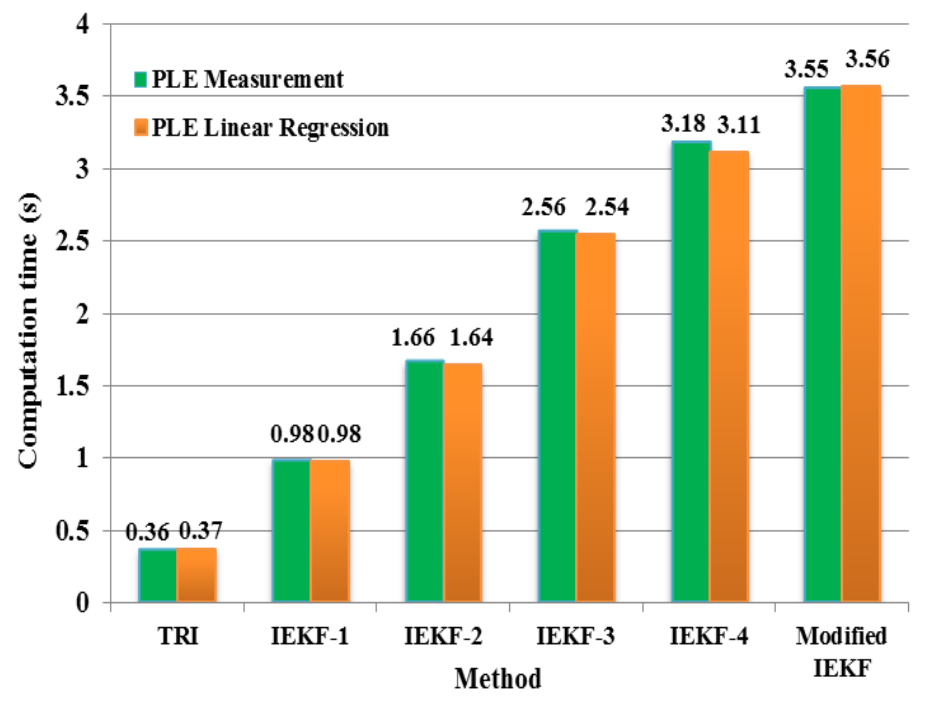

Fig. 15 Bar chart of computation time system

In otherwise when we tried for adding the iterations number to the 5 iterations, the computation time was took up to 4.05 seconds. It proves that computation time for modified IEKF is better than 5 iterations process although 5 iterations process has the small capability to the improve the error estimation. In this system, there are some correlations between error estimation, accuracy level and computation time. The best performance will be achieved when this system has low error estimation, high accuracy level and fast computation time. However, in this system, low error estimation and high accuracy level have long computation time. This condition can be occurred due to the combination of some algorithms. The computation time of this system is still better than adding the number iterations to reduce the estimation error. 
4) Comparison Analysis Based on Reference Node Number

The anchor node number which was used for obtaining the position estimation also influenced to this system. The position calculation from a distance based on RSSI data can be conducted at least using 3 reference node or anchor node. More than 3 references node is called as multilateration method. As shown in Fig. 16, there is 3 types estimation process based on reference number. Trilateration which is used 3 references node is compared with multilateration at 4 references node and 5 references node.

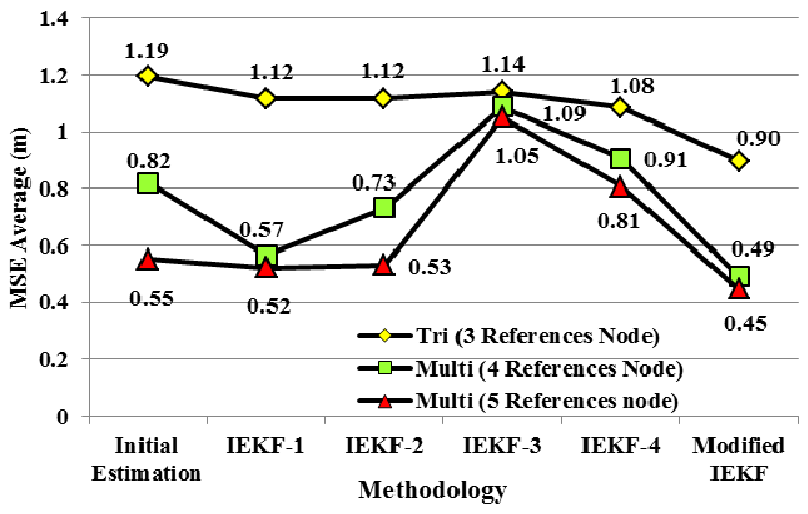

Fig. 163 types estimation process based on reference number

The result show, using more references node for estimating the mobile node has lowest MSE data 5.55 meters in the initial estimation process. Due to the fluctuative reduction from each IEKF iterations, adding modified IEKF is achieved better MSE performance up to 0.45 meter in using 5 references nodes. While using 4 references node have lower error performance at 0.49 meters which is better than using 3 references node. However, using many references node will increase the complexity of the process in IEKF algorithm. Many references node also influenced to the transmission data such as interference problem, as shown in [28]. Complexity process and transmission problem will influence this system when this system is implemented to the real hardware and real scenario.

In the previous related research, using cricket localization scheme based on ultrasound estimation was applied to the tracking system. The combination of trilateration and EKF algorithm for $\mathrm{P}$ model, PV model, and PVA model have been evaluated in [4]. The experimental result at the $2 \times 2$ meters observation area shows $0.42 \mathrm{~m}$ RMSE system for EKF P-model. This result is better than our proposed result which was achieved $0.88 \mathrm{~m}$ MSE or $0.93 \mathrm{~m}$ in the RMSE system. Our proposed system was applied to $54 \mathrm{~m} \times 57.4 \mathrm{~m}$ observation area which were larger than it. Fluctuative and varying data of RSSI measurement from Xbee-S2 (Pro) was influenced by our system. However, using RSSI measurement system have inexpensive and easier positioning method when it was implemented in large observation area which was required many numbers of node deployment.

An indoor tracking system based on inertial observations and RSSI measurements from a WSN have been proposed in [1]. It is common in hybrid positioning systems that combine RSSI and inertial measurements by means of an EKF algorithm to be accurate.The results show an RMSE of 1.5 meters at $11.2 \mathrm{~m} \mathrm{x} 47.6 \mathrm{~m}$ which were larger than RMSE $0.93 \mathrm{~m}$ our proposed system. Using only RSSI parameter measurements can be performed accurately to our mobile cooperative tracking system.

The performance of the newly proposed EKF technique, with bounding constraints on NLOS conditions, exceeds that EKF by a significant margin has been presented in [14]. A hybrid of TDOA and AOA measurements was used for tracking asynchronous targets of this system. The RMSE performance result showed at $0.5 \mathrm{~m} / \mathrm{s}$ which was better than our proposed method.

An iterative formulation of ensemble Kalman filter (EnKF) for the strongly nonlinear system is the perfect model framework. A simple modification which was turned the scheme into an ensemble formulation of the iterative EKF algorithm has been introduced in [24]. The RMSE performance of IenKF an IEKF of this system were $0.48 \mathrm{~m}$ and $0.60 \mathrm{~m}$ that took up to 10 iteration numbers. Compared to the previous related work, our proposed system can be performed fastly in computation time which was required only 4 iterations number. While according to the accuracy level than using TDOA or AOA measurement system, our proposed system can be reduced the error effectively although can't be strongly reduced as the TDOA or AOA.

\section{CONCLUSIONS}

In this paper, we propose mobile cooperative tracking scenario using modified IEKF based on its iterations. The cooperative tracking scenario is obtained from an effective distance of RSSI measurement phase with Xbee-PRO (S2) module. There are 4 estimation processes in this system. The first estimation is obtained from trilateration method according to the 3 nearest distance reference. EKF algorithm as the second estimation calculation is produced to refine the initial estimation. The output from EKF algorithm is processed to the IEKF algorithm as the initial iterations. This system takes 4 iterations process to reduce error estimation of mobile node position. Stability data of the IEKF algorithm from each iteration require addition algorithm to improve the accuracy level of this system. Using selection process based on average estimation value can modify the output of IEKF algorithm. The comparative analysis shows that modified IEKF algorithm for mobile cooperative tracking could decrease MSE of estimation position up to 0.5 times better than conventional IEKF algorithm and up to 2 times better than EKF algorithm. However, adding modified IEKF to the mobile cooperative tracking requires 0.4 seconds longer computation time than conventional IEKF which is faster than adding more iterations number.

\section{REFERENCES}

[1] A.Correa, Marc B., Antoni M. and J. L. Vicario, "Distance-based Tuning of the EKF for Indoor Positioning in WSNs", 22nd EUSIPCO, 1-5 September 2014.

[2] Bouchra El Madani, Anne Paule Yao, and Abdelouahid Lyhyaoui, "Combining Kalman Filtering with Zigbee Protocol to Improve Localization in Wireless Sensor Network", Hindawi Publishing Corporation ISRN Sensor Networks, vol.2013, 7 pages, 22 January 2013

[3] Sandy Mahfouz, Farah Mourad-Chehade, Paul Honeine, Joumana Farah, and Hichem Snoussi, "Target tracking using machine learning 
and Kalman filter in wireless sensor networks", IEEE Sensors Journal, vol.14, issue:10, pp:3715-3725, October 2014.

[4] Rafiullah Khan, Sarmad Ullah Khan Shahid Khan, and M. Usman Ali Khan, "Localization Performance Evaluation of Extended Kalman Filter in Wireless Sensors Network", ANT international conference Procedia computer science, pp:117-124, 2014.

[5] Gary Cullen, Kevin Curran, Jose Santos, Gearoid Marguire, and Denis Bourne, "CAPTURE-Cooperatively Applied Positioning Techniques Utilizing Range Extensions", International Conference on Indoor positioning and Indoor navigation, pp:340-346, 27-30 October 2014.

[6] Gary Cullen, Kevin Curran, and Jose Santos, "Cooperatively Extending the Range of Indoor Localization", ISCC 2013, pp:1-8, 20-21 June 2013.

[7] Rafina Destiarti, Prima Kristalina, and Amang Sudarsono, "Mobile Cooperative Tracking with RSSI Ranging in EKF Algorithm for Indoor Wireless Sensor Network", 5th KCIC, 15-17 November 2016.

[8] Prima Kristalina, Wirawan, and Gamantyo Hendrantoro, "Node Localization Methods with Improved Accuracy for Wireless Sensor Network", TENCON, pp: 535-539, 21-24 Nov 2013.

[9] Chin-Der Wann, "Kalman Filtering NLOS Mitigation and Target Tracking in Indoor Wireless Environment", INTECH, pp:309-326, May 2010.

[10] Chuan C.P., Soo Young L., and Pei Cheng O., "Measurement Arrangement for Estimation of Path Loss Exponent in Wireless Sensor Network", 7th ICCCT, pp: 807-812, 2012.

[11] J. Havlik and O. Straka, "Performance Evaluation of Iterated Extended Kalman Filter with Variable Step-length", 12th European Workshop on Advanced Control and Diagnosis (ACD), 2015

[12] Bo He, et all, "Simultaneous Localization and Mapping with Iterative Sparse Extended Information Filter for Autonomous Vehicles", Sensors Journal 2015, pp:19852-19879, 13 August 2015

[13] T. Sathyan, and Mark H. "Fast and Accurate Cooperative Tracking in Wireless Networks", IEEE Transactions on Mobile Computing, vol.12, No.9, September 2013

[14] S. Yousefi, Xiao-Wen C., and Benoit C., "An Improved Extended Kalman Filter for Localization of a Mobile Node with NLOS Anchor", 9th ICWMC, 2013.

[15] Laith K., and Peter Jung, "Scaled Unscented Kalman Filter for RSSIBased Indoor Positioning and Tracking", 9th Internationa Conference on Next Generation Mobile Applications, Services, and Technologies, 2015.

[16] Rafina D., Prima K., Amang S. "Cluster-Based PLE Areas for Mobile Cooperative Localization in Indoor Wireless Sensor
Network", International Conference on Information Technology and Electrical Engineering (ICITEE), October 2016, pp: 112-117.

[17] Moez Ben K., Alexandre J.R., Francois G., G. Gagnon, and Philippe L., "RSSI-based Indoor Tracking using the Extended Kalman Filter and Circulary Polarized Antennas", 11th Workshop on Positioning, Navigation and Communication (WPNC), 12-13 March 2014.

[18] Yuan $\mathrm{Xu}$, Xiyuan Chen, and Qinghua Li, "Autonomous Integrated Navigation for Indoor Robots Utilizing On-Line Iterated Extended Rauch-Tung-Striebel Smoothing”, Sensors Journal 2013, pp:1593715953, 25 November 2013.

[19] Li Liang-qun, Ji Hong-bing, and Luo Jun-hui, "The Iterated Extended Kalman Particle Filter", ISCIT 2005, 12-14 October 2005.

[20] K. Vadivukkarasi R. Kumar, and Mary Joe, "A Real Time RSSI Based Novel Algorithm to Improve Indoor Localization Accuracy for Target Tracking in Wireless Sensor Networks", ARPN Journal of Engineering and Applied Sciences, vol.10, no.16, September 2015.

[21] Liping Zhang, Cheng-chew Lim, Yiping Chen, nd Hamid Reza Karimi, "Tracking Mobile Robot in Indoor Wireless Sensor Networks", Hindawi Publishing Corporation Mathematical Problems in Engineering, vol.2014, 8 pages, 20 February 2014

[22] Seoung-Hyeon Lee, Il-Kwan Lim, and Jae-Kwang Lee, "Method for Improving Indoor Position Accuracy Using Extended Kalman Filter", Hindawi Publishing system Mobile information system, vol.2016, 15 pages, 14 June 2016.

[23] Yasmine K. B., Mokhtar K, and Karim Abed-Meraim, "Divided Difference Kalman Filter for Indoor Mobile Localization", 2013 International Conference on Indoor Positioning and Indoor navigation, pp:1-8, 28-31 October 2013.

[24] Pavel Sakov, Dean S. O., and Laurent B., "An Iterative EnKF for Strongly Nonlinear Systems", American Meteorological Society Journal 2012, pp:1988-2004. 1 June 2012.

[25] Jie Chen, "RSSI-based Indoor Mobile Localization in Wireless Sensor Network", International Journal of Digital Content Technology and its Applications, vol.5, no.5, pp:408-416, July 2011.

[26] B.R. Jadhavar, T.R. Sontakke, " 2.4 GHz Propagation Prediction Models for Indoor Wireless Communications Within Building", IJSCE, vol. 2, issue: 3, pp: 108-113, July 2012.

[27] Scott Y., Rappaport, "914 MHZ Path Loss Prediction Models for Indoor Wireless Communications in Multifloored Buildings", IEEE Antenna and Propagation, vol.40, pp:207-217, February 1992.

[28] Wail M., Yaser K., Reem J., and Rana H., "Interference Problem between Zigbee and Wifi”, IPCSIT, vol. 30, 2012. 